\title{
Permalloy thin-film magnetic sensors
}

\author{
J P J Groenland, C J M Eıkel, J H J Fluitman and R M de Ridder \\ University of Twente, MESA Research Institute, Faculty of Electrical Engineering. PO Box 217, NL-7500 AE Enschede (Netherlands)
}

\begin{abstract}
An introduction to the theory of the anısotropic magnetoresistance effect in ferromagnetic thın films is given, ending in a treatment of the minimalization of the free energy which is the result of the intrinsic and extrinsic anisotropies of the thin-film structure The anisotropic magnetoresistance effect in long strips is reviewed Attention is given to problems like the formation of domains and measures like biasing and linearization The paper concludes with a description of some applications which are being developed by the authors (1) an analyser for the stray field of recording heads, (2) a sensitıve magnetometer, (3) an accurate absolute angle detector, and (4) an absolute (linear) position detector
\end{abstract}

\section{Introduction}

Electrically conductive magnetic materials may show the anisotropic magnetoresistance effect, which means that the electrical resistivity depends on the direction of the magnetization vector If one considers a magnetic thin film, this effect results in resistivities which are different when measured perpendicular $\left(\rho_{\perp}\right)$ or parallel $\left(\rho_{\|}\right)$to the local magnetization direction If the magnetization in the film is determined by an external magnetic field, the thin-film device can act as a sensor for this field Then, a two-step process is acting in such a sensor a magnetic field determines the magnetization and the magnetization determines the resistivity A sensor for magnetic fields can be applied to detect position-, angle- or tıme-dependent information, since magnetic fields can be given a geometrical and/or temporal structure

In addition to the resistance anisotropy mentıoned above, magnetic bodies may possess magnetic anisotropy, which can be of intrinsic (material property) or extrinsic (shape effect) nature In a magnetic thin film, which does not have intrinsic anisotropy perpendicular to the film plane, the magnetization vector will be restricted to lie in the plane of the film to a very good approximation This is a consequence of the demagnetizing effect, which strongly reduces the magnetization component in a direction perpendicular to the film surface For this reason the description of the effect can be restricted to two dimensions, with only the inplane components of the external fields being effective in practical devices

It must be noted that the sensitivity of anisotropic magnetoresistance devices for in-plane magnetic fields is in contrast to that of normal magnetoresistive or Hall devices, which react to perpendicular field components In principle, the normal effect is also present in a magnetic conductor, but in the materials treated here it can be neglected with respect to the anisotropic effect

\section{The resistance anisotropy}

The anisotropic magnetoresistance effect implies the electric resistivity change

$\Delta \rho=\rho_{\|}-\rho_{\perp} \neq 0$

This phenomenon is present in three-dimensional metals and alloys as a consequence of the anisotropic scattering of the conduction electrons $[1,2]$ Some characterıstic $\Delta \rho / \rho$ values are listed in Table 1 (we define $\rho=\left(\rho_{\|}+\rho_{\perp}\right) / 2$ )

The choice of a suitable sensor material not only depends on $\Delta \rho / \rho$ values (actually in the percent regime), but also on other properties like the magnetic properties and the sensitivity for temperature or stress These and other considerations have generally led to the choice of magnetostriction-free 
TABLE 1 Characteristıc bulk values for $\Delta \rho / \rho$ at room temperature

\begin{tabular}{ll}
\hline Material & $\Delta \rho / \rho(\%)$ \\
\hline $\mathrm{N}_{1_{70}} \mathrm{Co}_{030}$ & 66 \\
$\mathrm{~N}_{042} \mathrm{Fe}_{018}$ & 43 \\
$\mathrm{~N}_{099} \mathrm{Fe}_{001}$ & 27 \\
$\mathrm{~N}_{094} \mathrm{Mn}_{006}$ & 25 \\
$\mathrm{~N}_{094} \mathrm{Sn}_{006}$ & 18 \\
$\mathrm{~N}_{083} \mathrm{Pd}_{017}$ & 23 \\
$\mathrm{Fe}_{090} \mathrm{~V}_{010}$ & 13 \\
\hline
\end{tabular}

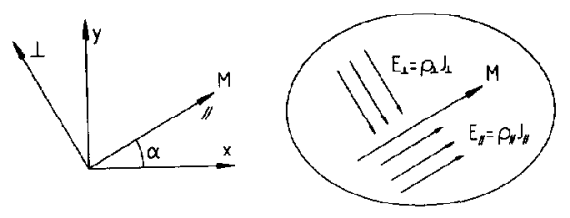

Fig 1 Magnetic thin film with magnetization vector $\boldsymbol{M}$ at an angle $\alpha$ with respect to the $x$ axis Currents are parallel or perpendicular to $M$ (see resıstıvities $\rho_{\mid}$and $\rho_{\perp}$ respectively)

permalloy $\mathrm{N}_{\mathrm{B}_{82}} \mathrm{Fe}_{0}{ }_{18}$ and, to a much lesser extent, $\mathrm{N}_{1} \mathrm{Co}$ and $\mathrm{N} 1 \mathrm{FeCo}$ alloys

Consider a resistance anisotropy induced by the magnetization vector, with no other anisotropy effects acting on the resistance present (the use of polycrystallıne thin films elımınates the crystallographic contribution by averaging) Under the assumption of a pure anisotropic magnetoresistance effect, Ohm's law can be written in tensor form

$\left(\begin{array}{l}E_{\|} \\ E_{\perp}\end{array}\right)=\left(\begin{array}{cc}\rho_{\|} & 0 \\ 0 & \rho_{\perp}\end{array}\right)\left(\begin{array}{l}J_{\|} \\ J_{\perp}\end{array}\right)$

where one of the coordinate axes is chosen along the magnetization vector ( $F_{1}$ 1) $E_{1}$ is the electric field strength and $J$ the current density Here $\rho_{\|}$ and $\rho_{\perp}$ are uniquely determined and do not depend on direction themselves

From a practical point of view it is more convenient to choose the $x$-axis of the coordinate system along $J$ so that only a component $J_{x}$ remains Transforming eqn (1) to that system results in

$$
\left(\begin{array}{c}
E_{\mathrm{r}} \\
E_{y}
\end{array}\right)=\left(\begin{array}{cc}
\rho+\frac{1}{2} \Delta \rho \cos 2 \alpha & \frac{1}{2} \Delta \rho \sin 2 \alpha \\
\frac{1}{2} \Delta \rho \sin 2 \alpha & \rho-\frac{1}{2} \Delta \rho \cos 2 \alpha
\end{array}\right)\left(\begin{array}{c}
J_{\mathrm{r}} \\
0
\end{array}\right)
$$

with $\alpha$ the angle between the magnetization vector $\boldsymbol{M}$ and the $x$-axis Equation (2) can also be written as

$E=\left(\begin{array}{c}\rho J_{r} \\ 0\end{array}\right)+\frac{1}{2} \Delta \rho J_{x}\left(\begin{array}{c}\cos 2 \alpha \\ \sin 2 \alpha\end{array}\right)$

It is easy to generalize eqn (3) and express the component $E_{\zeta}$ along a direction at an angle $\zeta$ with respect to the direction of $J$

$E_{\zeta}=\rho J \cos \zeta+\frac{1}{2} \Delta \rho J \cos (2 \alpha-\zeta)$

\section{Magnetization versus magnetic field}

Permalloy films are characterized by the occurrence of an intrinsic uniaxial magnetic anısotropy [3] This anisotropy can be specified by the energy density, which is necessary to disorient the magnetization vector $\boldsymbol{M}_{\mathrm{s}}$ with respect to the anisotropy direction The subscript $s$ indicates that in this type of film, the magnitude of the magnetization vector has a maximum, or saturation, value This energy term reads

$\varepsilon_{\mathrm{a}}=K \sin ^{2} \beta$

with $K$ the constant of uniaxial anisotropy and $\beta$ the angle between $\boldsymbol{M}_{\mathrm{s}}$ and the anisotropy axis (also called the easy axis), which we take along the $x$-axis (Fig 2) The strength of the anisotropy is often represented by the anisotropy field $H_{\mathrm{k} \text { intr }}$ (in the remainder of this paper often denoted as $H_{\mathrm{k}}$ ), defined as

$H_{\mathrm{k} \text { intr }}=2 K / \mu_{0} M_{\mathrm{s}}$

where $\mu_{0}$ is the permeability of free space The anisotropy is determined by film growth conditions and/or subsequent treatment (magnetic annealing) A characteristic value for the anisotropy field strength is $300 \mathrm{~A} / \mathrm{m}$

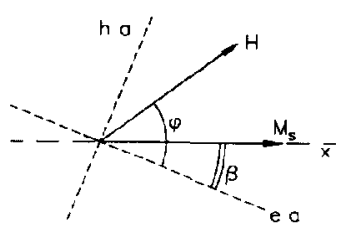

Fig 2 Definition of $\varphi$ and $\beta$ e a is easy axis, $\mathrm{h}$ a is hard axis 
Apart from the anisotropy, the direction of the magnetization vector $\boldsymbol{M}_{\mathrm{s}}$ is determined locally by the local magnetic field $\boldsymbol{H}$ If such a field is present, the expression for the free energy density reads

$\varepsilon=K \sin ^{2} \beta-\mu_{0} H \cdot M_{\mathrm{s}}$

The equilibrium value for the magnetization direction is determined by mınımızing eqn (7) with respect to $\beta$ Takıng

$\delta \varepsilon / \delta \beta=2 K \sin \beta \cos \beta-\mu_{0} M_{\mathrm{s}} H \sin (\varphi-\beta)=0$

it follows that

$\sin (\varphi-\beta)=H_{\mathrm{k}} \sin (2 \beta) / 2 H$

with $\varphi$ the angle of $\boldsymbol{H}$ with the anisotropy axis (Fig 2) Equation (8) must be solved under the condition $\delta^{2} \varepsilon / \delta \beta^{2} \geq 0$ Unfortunately this can be done in only a few special cases, although it can be visualized very elegantly with the help of the socalled Stoner-Wohlfarth astroid (Fig 3) With the help of this device stable solutions can be constructed via a geometrical procedure [4] Note that the procedure gives a single solution for an infinity of $\boldsymbol{H}$-vectors (e g, $M_{\mathrm{s}_{1}}$ is a solution for $H, H^{\prime}$ and $H^{\prime \prime}$ in Fig 3), which emphasizes the need for measures to design a device with a uniquely defined output

This Section concludes with three remarks The first concerns the anisotropy, which always has some spread in magnitude as well as in direction This is commonly denoted as dispersion

Furthermore, the easy-axis orientation is temperature and stress dependent [5] The second

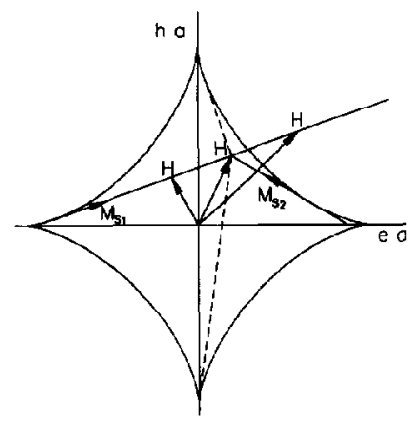

Fig 3 Stoner-Wohlfarth astroid Magnetization directions are constructed by taking the tangents from the arrow tup of $\boldsymbol{H}$ to the astrold in the direction of the easy axis remark concerns exchange interaction A contribution from this interaction to the energy is neglected in eqn (7) This is permitted as long as we consider a single-domain model, or magnetization distributions with relatively small gradients ('almost' single-domain model) In the practical configurations, which are the subject of subsequent Sections, this condition is always fulfilled [6] The third remark concerns the switching time In principle the coherent switching of magnetic dipoles is governed by intrinsic and eddy current damping, which is greatly reduced in thin films This makes very high switching rates (up to $1 \mathrm{GHz}$ ) possible [3]

\section{The anisotropic magnetoresistance effect in (long) strips}

This Section treats the strip depicted in Fig 4, which is considered to be very long with the current contacts far apart This is for two reasons First, we may expect the occurrence of domains at the end of the strip (see Section 5), while we want to confine ourselves to the 'almost' single-domain model Secondly, the current contacts have a short-circuiting effect on the transverse voltage (which we call the planar Hall voltage) in the vicinity of the current contacts This effect is extensively treated in the literature [7] for the normal Hall effect and has a comparable influence on the planar Hall effect [8] Therefore a further treatment is omitted here The influence of the short-circuiting effect on the (lateral) magnetoresistance is negligible in the planar case [9] This is in contrast to the normal magnetoresistance effect

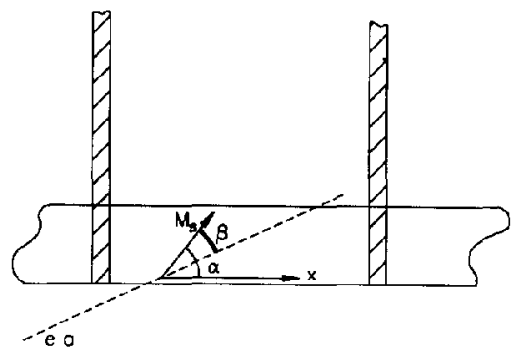

Fig 4 Long stnp configuration 
The expression for the free energy of a strip, neglecting end effects, is

$$
E=\int_{\text {vol }} K \sin ^{2} \beta-\frac{1}{2} \mu_{0} \int_{\text {vol }} M_{\mathrm{s}} \cdot H_{1}-\mu_{0} \int_{\text {vol }} \boldsymbol{M}_{\mathrm{s}} \cdot \boldsymbol{H}_{\mathrm{a}}
$$

$H_{\text {a }}$ is the externally applied field, while $H_{1}$ is the demagnetizing field, or (dipole-dipole) interaction field, generated by the divergence of $M$ The factor $\frac{1}{2}$ in the second right-hand term is a consequence of the mutuality of the dipole-dipole interaction [10], where one part of the device generates the field acting on another, and vice versa If, for mathematical convenience, a long strip with an elliptic cross section is considered, a homogeneous magnetization (single-domain film) leads to a constant value for $H_{1}$ at every position of the film

$H_{1}=-D M_{\mathrm{s}} \sin \alpha$

with $\alpha$ the angle between $\boldsymbol{M}_{\mathrm{s}}$ and the strip axis and $D$ the demagnetızing factor, for which $D=t /(t+w)$ is valid to a good approximation in this case ( $t$ and $w$ are the thickness and the width of the strip, respectively, with $t \ll w$, e g, $t=40 \mathrm{~nm}, w=4000 \mathrm{~nm}$ )

So we find for this case

$\varepsilon=\frac{E}{\text { vol }}=K \sin ^{2} \beta+\frac{1}{2} \mu_{0} D M_{\mathrm{s}}^{2} \sin ^{2} \alpha-\mu_{0} \boldsymbol{M}_{\mathrm{s}} \cdot \boldsymbol{H}_{\mathrm{a}}$

It is seen that the interaction term behaves formally as a uniaxial anisotropy along the strip axis (shape anisotropy) Although practical specimens (to which we shall return shortly) will not have an elliptical cross section, the assumption is well suited for first estımates

If $\alpha=\beta$ (coinciding anisotropy axes) an effective anisotropy $K^{*}$ can be defined

$K^{*}=K+\frac{1}{2} \mu_{0} D M_{\mathrm{s}}^{2}$

This leads to an 'effective' anısotropy field

$H_{\mathrm{k}}^{*}=H_{\mathrm{k} \text { intr }}+H_{\mathrm{k} \text { shape }}=H_{\mathrm{k} \text { intr }}+D M_{\mathrm{s}}$

Under the assumption of an elliptical cross section, the homogeneity of $\boldsymbol{M}$ is justified afterwards if $H_{a}$ is homogeneous as well If $H_{a}$ is directed perpendicular to the strip axis, it is convenient to introduce the normalized applied field $h_{\mathrm{a}}$ as
$h_{\mathrm{a}}=H_{\mathrm{a}} / H_{\mathrm{k}}^{*}$

and the single-domain model leads to

$\left.\begin{array}{ll}\sin \beta=h_{\mathrm{a}} & \text { for } h_{\mathrm{a}} \leq 1 \\ \sin \beta=1 & \text { for } h_{\mathrm{a}}>1\end{array}\right\}$

Equation (15) can easily be verified by minımızıng eqn (11) Introduction of eqn (15) into eqn (3) leads to

$E_{\mathrm{x}}=\rho_{\perp} J-\Delta \rho J h_{\mathrm{a}}^{2}$

$E_{v}=\Delta \rho J h_{\mathrm{a}}\left(1-h_{\mathrm{a}}^{2}\right)^{\frac{1}{2}}$

or, since $\beta$ is constant over the strip, to

$V_{\mathrm{MR}}=\imath R-\imath \Delta R h_{\mathrm{a}}^{2}$

$\frac{l}{w} V_{\mathrm{H}}=\imath \Delta R h_{\mathrm{a}}\left(1-h_{\mathrm{a}}^{2}\right)^{\frac{1}{2}}$

with $V_{M R}$ and $V_{H}$ the magnetoresistance and planar Hall voltages respectively, $l$ the sensor drive current, $R$ and $\Delta R$ the sensor resistance and maximum sensor-resistance change respectively and $l$ the sensor-strip length Equations (16) to (19) are valid for $\left|h_{\mathrm{a}}\right| \leq 1$ For larger values of $\left|h_{\mathrm{a}}\right|, h_{\mathrm{a}}$ should be replaced by 1 in these equations The expressions (18) and (19) have been plotted in Fig 5 (curves numbered 1 )

If $\alpha \neq \beta$ (non-coinciding anisotropy axes) an effective anisotropy can be defined in direction as well as in magnitude The results for this situation are not derived here, but can be found in the hiterature [11]

Returning to the more realistic non-elliptic cross section, a form effect may be expected as well, although a shape anisotropy cannot be specified exactly The assumption of a homogeneous

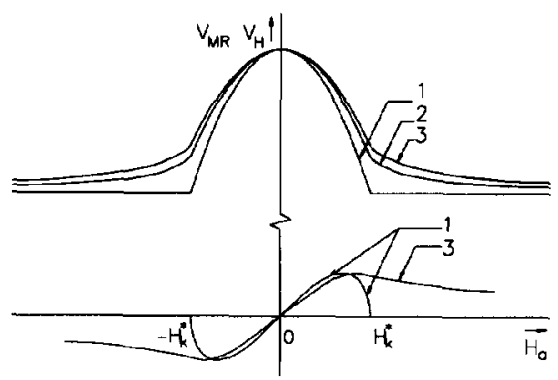

Fig $5 \quad V_{M R}$ (upper curves) and $V_{H}$ (lower curves) for a rectangular cross section with shape parameter $a_{3}=H_{\mathrm{k} \text { shape }} / H_{\mathrm{k}}^{*} \quad 1$, Instrinsic behaviour with $a_{5}=0$ (also valid for elliptic cross section), 2, $a_{\mathrm{s}}=0 \mathrm{5}$, $3, a_{\mathrm{s}}=1$ 


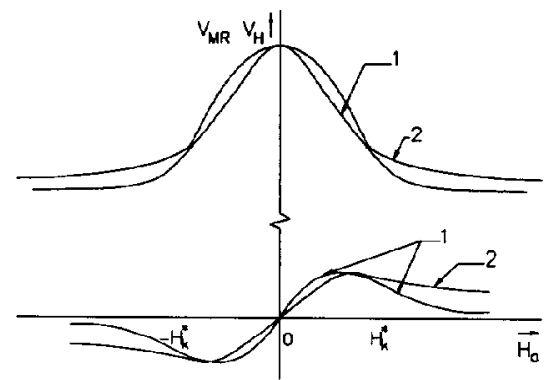

Fig 6 Influence of edge profile on magnetoresistance and planar Hall effect, curves of devices having the same shape parameter $a_{\mathrm{s}}=0095$ 1, edge tapered over $70 \%$ of strip width, 2 , rectangular profile

magnetization no longer leads to a constant value for $H_{1}$, which in turn makes the assumption of a homogeneous $\boldsymbol{M}$ no longer justified Nevertheless, the problem can be solved with the help of numercal procedures using a computer We shall not go into the details of such computations, which are given elsewhere [12], but only present some characteristic results of $V_{\mathrm{H}}$ and $V_{\mathrm{MR}}$ curves for a number of ratios $a_{\mathrm{s}}=H_{\mathrm{k} \text { shape }} / H_{\mathrm{k}}^{*}\left(\mathrm{~F}_{1} \mathrm{~g}\right.$ 5) Again the intrinsic anisotropy is assumed to be parallel to the strip axis while the applied field is perpendicular to it

In contrast with the 'ellıptıc' case, the shape of the curves depends on the aspect ratio of the strip and it can be seen that the assumption of an elliptical cross section may lead to rather larger deviations for $a_{\mathrm{s}} \approx 1$ This raises the supposition that in this limit any deviation from rectangularity may lead to a significant deviation from the results of Fig 5 This can easily be shown from a computation in which an edge-taper is assumed (Fig 6) [12] Experimental curves on specimens with a large form effect often show deviations which can easily be ascribed to specific edge profiles, poor edge definition, edge asperities and so on

\section{Domains $[3,13 \mid$}

In Section 4 only (almost) single-domain solutions were considered No attention was paid to solutions that contain magnetic domains, which may easily occur in practice

Th1s can be demonstrated by the examples of Fig 7 A magnetic field in a direction perpendicular to the strip axis with a magnitude larger than

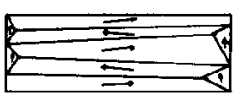

(a)

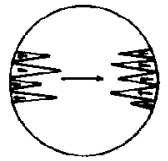

(b)
Fig 7 Possible domain configurations (a) in a short strip after relaxation from a large field perpendicular to the strip axis, (b) in a disc after relaxation from a large field pointing to the night In both cases the easy axis is horizontal

$H_{\mathrm{k}}^{*}$ pulls the magnetization perpendicular to the strip axis If such a field decreases and becomes smaller than $H_{\mathrm{k}}^{*}$ again, the magnetization will start to rotate back to the easy-axis position However, the rotation may be clockwise or ant1-clockwise and, depending on the local deviations from the anisotropy direction (angular dispersion), the magnetization will rotate in opposite directions in different parts of the film This results in a multidomain film with (approximately) opposite magnetization directions when the external field has diminished to zero (F1g $7(\mathrm{a})$ )

A second example of domain formation can be given on the basis of the situation in discs or short strips Starting from a decreasing but positively oriented magnetic field, the magnetization will switch from positive to negative at $H=-H_{\mathrm{k}}^{*}$ if the Stoner-Wohlfarth (SW) model is strictly followed In practice, however, the switching process starts at smaller (absolute) values of $H$ as a consequence of domain formation, often starting at the film edges So, instead of switching coherently, as predicted by the SW model, the film 'switches' by domains that grow in opposite directions throughout the film (Fig 7(b)) [3] This gives the switch a noisy appearance, resulting in Barkhausen noise in the electrical output

\section{Biasing and linearization}

Switching of the magnetization, whether or not accompanied by domain formation and growth, should generally be avoided in a working device In practice this means that the fields to be detected must be on a trajectory outside the SW astroid Such a requirement cannot be fulfilled if the device serves as a detector of unknown (possibly small) fields However, in applications where permanent 
magnets are part of the device (e $\mathrm{g}$, in position and angle detectors), such a requirement can be fulfilled (see applications described in Sections 73 and 74 )

When small fields in a limited range should be detected ( $\mathrm{e} g$, by a recording read head) a bias field may be added to shift the range outside the switching region, so that all events take place on a single branch of the magnetoresistance or planar Hall curve Apart from this, a bias field can be used to put a small-amplitude signal around a point of maximum sensitivity [14]

In most applications the use of the magnetoresistive effect in long strips is preferred over the use of the planar Hall effect, although the latter is linear in first order The reason for this is simple and can be derived from eqns (18) and (19) In order to make the domain influence and the short-circuiting effect at the strip ends negligible, a large $l / w$ ratio is required, leading to a large ratio of the magnetoresistive to the planar Hall voltages

Since in many cases the quadratic magnetoresistor response (eqn (18)) is not desirable, there is a need for linearization Avallable methods have been reviewed by Tsang [15] and Kwlatkowsk1 and Tumanskı [16] From the combination of eqns (3) and (15), it can be seen that a (firstorder) linear magnetoresistive response is obtained if the direction of the current flow is oriented at $45^{\circ}$ with respect to the easy axis $\left(1 \mathrm{e}, \alpha-\beta=45^{\circ}\right.$ in Fig 4), while still maintaining the easy axis oriented perpendicular to the magnetic field to be measured An important variation on this theme is the so-called barberpole configuration [17], where the edsy axis of a magnetoresistive strip is oriented parallel to its main geometrical axis, but where a highly conductive overlay structure deposited on the strip forces the current to flow locally at $45^{\circ}$ to the strip axis In a different approach to linearizatıon, a magnetic bias field can be applied ( $\mathrm{e} g$, [18-20])

\section{Applications}

An1sotrop1c magnetoresistive devices are used to measure magnetic fields and all variables that can exploit a magnetic field as an intermediate agent, e $\mathrm{g}$, angular position, linear position, temperature, etc Besides, they are widely used and investigated as detectors for magnetically stored information, $\mathrm{e} g$, in disc drives or in bubble memories In particular the application as a read head for magnetic recording (suggested by Hunt [21]) has been a subject of permanent investigation The advantage of a magnetoresistive (MR) head over a conventional inductive head is that it scales down favourably (with respect to the latter) regarding the signal-to-noise ratio [22] The obvious drawback of the MR head is that it must be combined with a separate write head Nowadays the MR read head is in use in some advanced recording systems and its role in magnetic recording can hardly be circumvented We do not go into the details of the recording MR head in this review, but refer the reader to the abundance of literature on the subject

\section{Field analyser}

A magnetoresistive permalloy strip can be used in a measuring system for the purpose of mapping the fringing fields of recording heads (including ring-type ferrite heads, thin-film heads and singlepole-type heads for perpendicular recording) and the flux reversals in hard-magnetic layers [23, 24] Depending on the position of the transducer with respect to the head or magnetic-layer surface, the perpendicular or tangential field component is measured (Fig 8)

The transducer is designed such that the magnitude of the field to be measured is smaller than $H_{\mathrm{k}}^{*}$, but of comparable order This can be done by a proper choice of the aspect ratio $t / w$ Since the detector output is not uniquely determined by an external field (see Fig 3), use is made of the knowledge that the nature of the fringing fields is two dimensional and the strip axis is positioned in the direction of the zero component of the magnetic-field pattern In measuring a recording-head field, a possible disturbing field along the strip axis will generally be screened out by the soft-magnetic material of the head itself and does not influence the sensor's signal

The magnetic-field component can be measured directly if there is a negligible field gradient along the width of the sensor strip This is generally not the case, because the head-gap length may be of the order of one micrometer so that significant field changes occur within sub-micron distances In that case the sensor signal has to be compared with computed values which have been derived on 


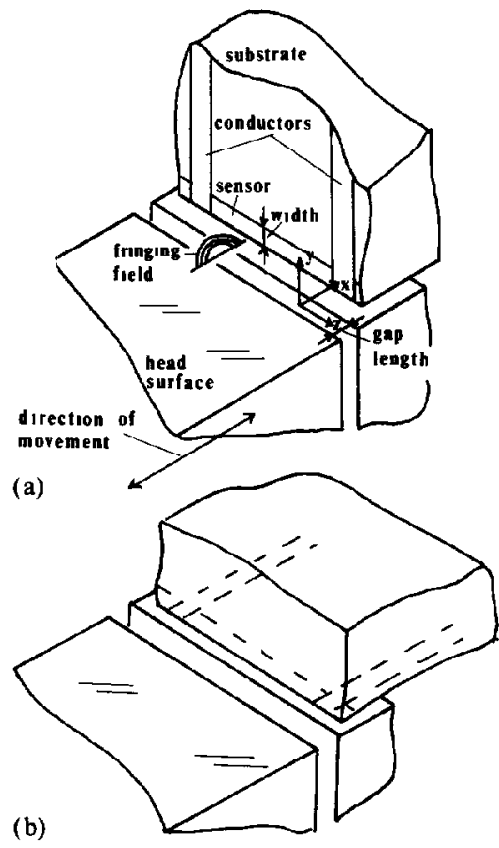

Fig 8 Orientation of the sensor substrate with respect to a nng-type head in order to determine the $y$ component (a) or the $x$ component (b) of the frning field

the basis of an assumed structure of the ficld and a model of the behaviour of a permalloy sensor strip in a non-uniform field [25] The character of this type of measurement requires an unbiased detector However, if the fringing field of a flux reversal in a hard-magnetic layer is measured, a linearization of the sensor is possible by means of an externally applied homogeneous field in the width direction of the sensor strip [24]

The technology of the preparation of permalloy transducers with micron or sub-micron width d1mensions on the very edge of a substrate can be found in the literature $[26,27]$

\section{Thin-film magnetometer}

In most designs of magnetoresistive magneticfield sensors, the occurrence of magnetic domains is detrimental to their operation In the magnetometer described here, which 1 s based on an inductive thin-film magnetometer first reported by West et al [28], the occurrence of magnetic domains is exploited for obtaining a sensitive magnetic-field sensor It has been shown $[29,30]$ that for low operating frequencies and small film dimensions, magnetoresistive rather than inductive readout of the magnetic state of the film will in principle lead to a better sensitivity An alternatıng (e g, sinusoidal) bias field $H_{\text {ex }}$ is applied perpendicular to the measurand and to the easy axis of a magnetoresistive film which is shaped into a planar Hall element or a magnetoresistor having a canted current path The bias field periodically saturates the film, and upon returning from saturation the magnetization splits into two parts (distributed over a number of magnetic domains) which rotate toward the easy axis in opposite directions The easy-axis field component, $H_{\mathrm{s}}$, determines the ratio of the two magnetization fractions, which in turn determines the sensor's output voltage Using an orthogonal susceptibility model [31] and neglecting dispersion in the magnitude of anisotropy and shape effects, the peak-to-peak output voltage $V_{\text {ss }}$ for a planar Hall configuration (shown in Fig 9) can be calculated to be $[30,32]$

$V_{\mathrm{ss}}=\frac{2 l w \Delta R}{\sigma_{\mathrm{a}} H_{\mathrm{k}} l \sqrt{2 \pi}} H_{\mathrm{s}}$

where $\sigma_{\mathrm{a}}$ is the standard deviation of the distribution function of anisotropy-axis onentations (angular dispersion) and $H_{\mathrm{s}}$ is the measurand This result is independent of the amplitude of $H_{\mathrm{ex}}$, which should, of course, be larger than $H_{\mathrm{k}}$ The waveforms occurring with a sinusoidal excitation field are shown in Fig 10

A small hysteresis effect in the sensor can be explained by the dispersion in the magnitude of anisotropy and the inhomogeneous demagnetizing field due to the non-elliptic cross section of the permalloy film [32]

The feasibility of fabricating this type of magnetometer with an integrated drive coll using planar technology (Fig 11) has been demonstrated [33]

If appropriate driving and signal-processing electronics are employed (using a non-sinusoidal

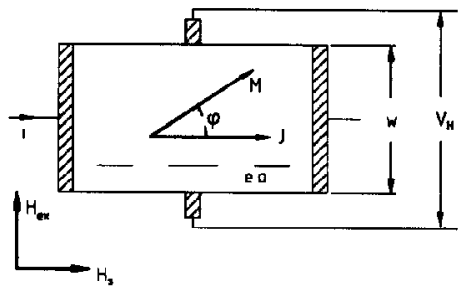

Fig 9 Magnetometer configuration, using the planar Hall effect $H_{\mathrm{ex}}$ is the periodical excitation field, $H_{\mathrm{s}}$ is the magnetic field component to be measured 


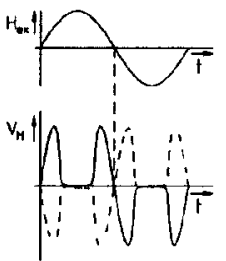

(a)

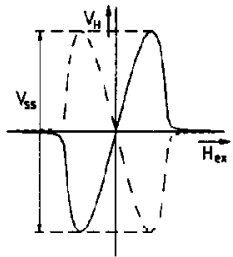

(b)

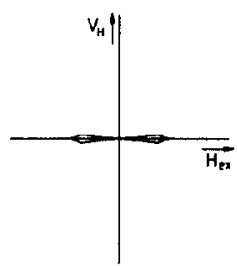

(c)
Fig 10 Magnetometer operation (a) $H_{\mathrm{ex}}$ and $V_{\mathrm{H}}$ vs time, (b), (c) $V_{\mathrm{H}}$ vs $H_{\text {ex }}$ (a) and (b) solid curve, $H_{\mathrm{s}}=H_{\mathrm{s} 0} \neq 0$, dashed curve $H_{\mathrm{s}}=-H_{\mathrm{s} 0}$ (c) $H_{\mathrm{s}}=0$

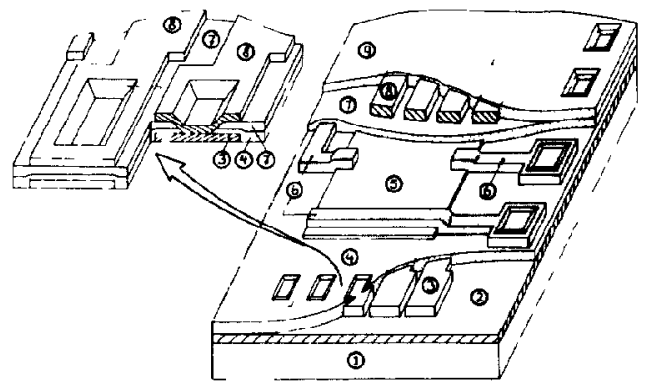

Fig 11 Magnetometer construction (1) $\mathrm{S}_{1}$ substrate, (2) $\mathrm{S}_{1} \mathrm{O}_{2}$ insulator, (3) Al lower half of excitation coll, (4), (7), (9) polyımide insulator, (5) permalloy, (6) Al contacts to permalloy, (8) Al upper half of coll (drawing by Rineke Groothengel)

excitation field), two magnetic-field sensors of this type can be applied in a solid-state compass, measuring both field components in the plane of the film with an accuracy of $3 \times 10^{-2} \mathrm{~A} / \mathrm{m}$ [30] This would allow the orientation of the earth's magnetic field to be measured with an accuracy of approximately $1^{\circ}$

\section{An absolute angle detector}

Whereas many applications of magnetoresistive films use relatively small magnetic fields, there are examples of magnetoresistive sensors which use very large (with respect to $H_{\mathrm{k}}^{*}$ ), so-called 'saturating', magnetic fields In such a sensor, all problems related to the formation of magnetic domains, to hysteresis, etc are avoided In homogeneous saturatıng fields, the performance of magnetoresistive elements can be optimized with respect to the signal-voltage level [34]

One example is an absolute angle detector $[35,36]$, shown schematically in Fig 12 A pair of magnetoresistive elements is used to detect the orientation of the (saturating) magnetic field of a

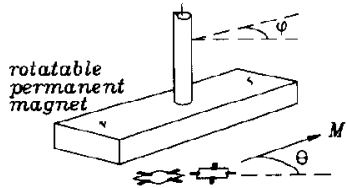

F1g 12 A contactless absolute angle detector The ortentation of the permanent magnet with respect to the magnetoresistive elements can be measured using the output signals of the elements $M$ represents the magnetization in the magnetoresistive elements

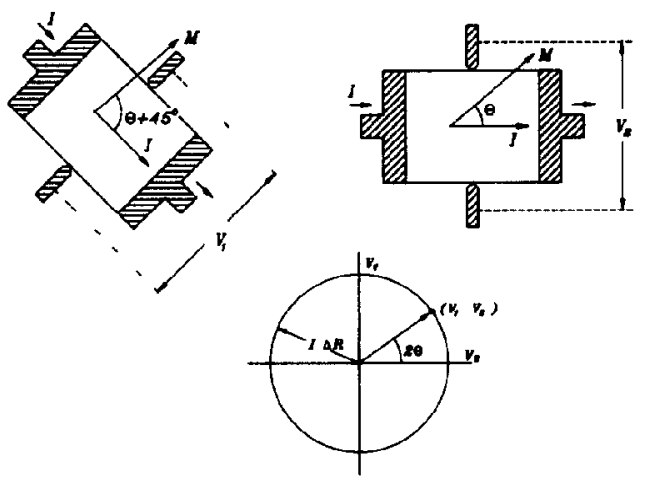

Fig 13 A pair of planar Hall elements as used in the detector of Fig $12 I$ represents the driving current, $M$ the magnetization of the permalloy A two-dimensional plot of $V_{1}=I \Delta R \cos (2 \Theta)$ and $V_{2}=I \Delta R \sin (2 \theta)$ is also shown

permanent magnet Magnet and magnetoresistive elements do not necessarily have to be joined mechanically, as long as the orientation of the magnetic field with respect to the magnetoresistive elements represents the angle that is to be measured This allows some freedom in the positioning of the magnet with respect to the elements, if the magnetic field is homogeneous in a certain area

The magnetoresistive elements are planar Hall elements (Fig 13), mutually rotated over 45 degrees The output angle $\Theta$ can be calculated from the two signals from these elements In Fig 13 the two signal voltages are plotted in a two-dimensional diagram for different values of $\Theta$ It is obvious that the sensitivity of this measurement is constant in the range of the angle detector 0 to 180 degrees Furthermore, the measurement is intrinsically temperature independent a change in temperature will affect $\Delta \rho$, and will cause a change in the radius of the circle in Fig 13 The measured value of $\Theta$, however, will not be affected 
In Fig 13 the short-circuiting effect of the current terminals of the elements is neglected This effect does not change the principle of the measurement, since it can be represented by a constant multiplication factor in $V_{1}$ and $V_{2}$

The magnetic anisotropy of the permalloy film causes a difference in orientation of the magnetic field and the magnetization in the film This effect causes a systematic error in the sensor signal Although this error can be accounted for in the electronics following the angle detector, it would be more convenient to use a permalloy film without magnetic anisotropy This can be realized by an annealing treatment of the permalloy film [35] or by using a double layer of two permalloy films with mutually perpendicular magnetic anisotropies [37]

Several solutions for readout electronics have been tested The signal of the detector is very similar to that of a resolver Therefore, standard resolver electronics can be used $O n$ the other hand, the signal processing of the angle detector is much more flexible than that of a resolver (a c or d c excitation currents, two independent elements), allowing a number of simpler solutions to be exploited

Another angle detector that operates in a saturatıng magnetic field is shown in Fig 14 This Figure shows a four-pole system, but the number of poles can be increased, if desired The signal of this type of sensor has a periodicity of $n$ if the magnet consists of $n$ poles Due to the symmetry of the system, the signal voltage $V_{\text {ab }}$ is relatively insensitive to misplacement of poles on the magnet, or misplacement of the magnet from the centre of the permalloy ring pair $A$ possible application of

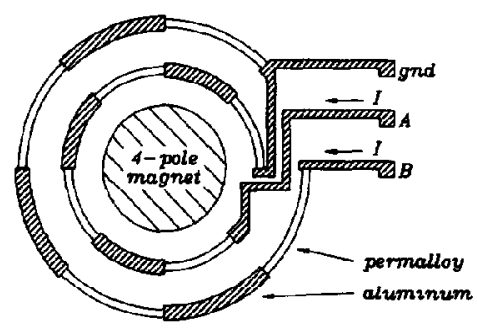

Fig 14 A four-pole detector A pair of permalloy nngs, which are partly covered with aluminium, is magnetized by the field of the magnet The rings are driven with a current $I$ In this configuration, an offset-free signal $V_{a b}$ is obtained, which is approximately sinusoidal this type of angle detector is to replace the collector in a $\mathrm{d} c$ motor

Depending on the position of the rotor, the current through the driving coils in the motor can be switched with the aid of the signals from the detector Preferably, zero-crossings of the signal are used Different phase shifts of the signal can be obtained from extra ring pairs which are slightly rotated with respect to the ring pair shown in the Figure The signal, which is approximately sinusoidal, can easily be transformed into the switching information for the driving coils and other interesting information like the speed of the rotor can easily be obtained A practical drive application of this sensor is currently being prepared for publication [38]

\section{An absolute position detector}

A system for the absolute detection of linear (or angular) position can be realized using a magnetoresistive permalloy strip as the position-data detector [39] In this system the position information is not conventionally land down in parallel tracks but is recorded in one single track of a hard-magnetic scale (see Fig 15) by means of a maxımumlength sequence of $2^{n}-1$ bits [40] The position coordinate can be derived from the response of a sensor array detecting a series of $n$ adjacent bits Maximally $2^{n}-1$ unique bit combinations can be distinguished

The magnetoresistive sensor array is built up of a permalloy strup land down on the edge of a substrate and is divided into separate sensor elements by means of electrical conductor strips perpendicular to the sensor strip The magnetization distribution in the permalloy strip is determined by

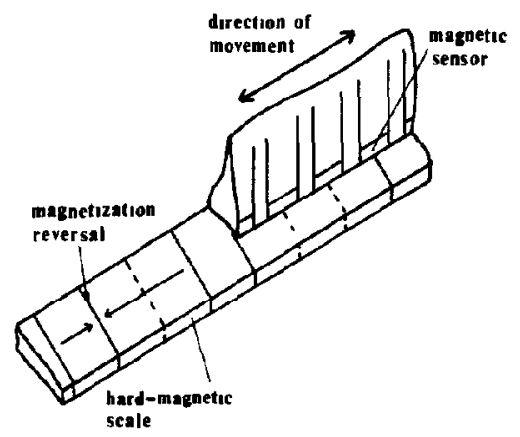

Fig 15 Detection of bits in a hard-magnetic layer by means of a magnetoresistive sensor array 


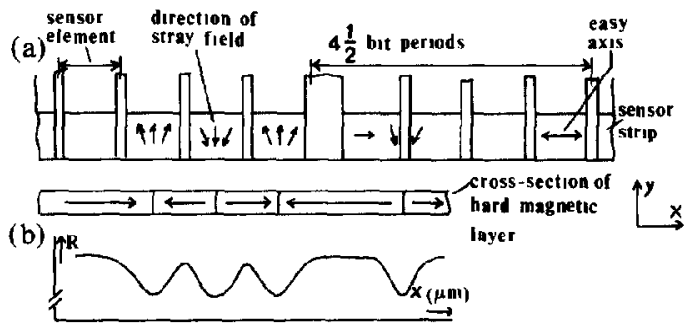

Fig 16 (a) Sensor strip, divided into two sets of sensor elements, positioned in the stray field of a hard-magnetic information layer (b) Output of a sensor element

the stray field of the hard-magnetic layer (Fig 16) The resistance of each sensor element is an indication for the presence (" $1-$ bit') or the absence (' 0 bit') of a flux reversal at that particular position in the scale The resistance values have to be corrected for the temperature effect of the magnetoresistive material by using dummy elements To avold ambiguities at the boundaries of the fluxreversal positions, at least two sets of sensor elements are necessary, which should be mutually shifted over $(n+1 / 2)$ bit periods (in the case of two sets) Some mechanism has to be created to decide on the choice between the two sets of sensor elements An obvious solution is the introduction of a second (reference) track with an all-ones recording pattern Such an additional track can also serve as the basis of an interpolation scheme for the purpose of improving the resolution of the position readout

Prototype detection heads have been realized with strip widths in the range of 10 to $30 \mu \mathrm{m}$ and $H_{\mathrm{k}}^{*}$ values of 1500 to $3600 \mathrm{~A} / \mathrm{m}$ The cross-contact leads, which must show a very low specific contact resistance to the permalloy film, were realized by patterning a Mo/Al film using a lift-off process The response of the sensor elements with various dimensions in the stray fields of the flux reversals of magnetic scales (actually commercially available audio tapes were chosen) was evaluated It was found that position bits can be detected with a lower bit-period limit of the order of a few hundred $\mu \mathrm{m}$

Based on experiments and on calculations with a simulation model, it can be concluded that the resolution of the position-detection system is of the order of $100 \mu \mathrm{m}$, which in principle can be improved by a factor of 10 to 100 with the application of interpolation techniques The performance of a system based on an alternative technique, being the semiconductor silicon Hall technology, is estimated to be of the same order as the permalloy-based version [41] The range of the position detector depends on the position-bit period and the number of applied elements in the sensor array and in principle is not limited

\section{Concluding remarks}

In the last few decades much work has been done concerning the theoretical description of anisotropic-magnetoresistance phenomena and the development of applications The commerical feasibility of this sensor type has been shown in the field of analog magnetic sensors and magneticrecording read heads, respectively However, the magnetoresistive sensor has not yet emerged as a low-cost mass-product One of the obstacles is the development of fabrication techniques for the realization of thin-film devices in large quantities, with good and reproducible properties and in a suitable package Another aspect is the integration with electronics in order to cope with special problems like temperature effects, initialization, biasing, drive currents and communications Multidisciplinary activities of this kınd are found to form a certain barrier for the utilization of these designs

\section{References}

1 T R McGuire and R I Potter, Anısotropic magnetoresistance in ferromagnetic 3d alloys, IEEE Trans Magn, MAG-11 (1975) $1018-1038$

$2 \mathrm{~J}$ W F Dorleujn, Electrical conduction in ferromagnetic metals, Phulps Res Rep 31, (1976) 287-408

3 R F Soohoo, Magnenc Thm Films, Harper and Row, New York 1965, p 93, p 159

$4 \mathrm{M} S$ Cohen, Ferromagnetism in films, in $\mathrm{K}$ L Chopra (ed), Thin Flm Phenomena, McGraw-Hill, New York, 1969, p 608

5 W H G Horsthuis, $K J \mathbf{M}$ Eijkel, $R \mathbf{M}$ de Ridder and $H$ Leeuwis. Thermally induced reversible easy axis rotations in permalloy thin films, Thin Sohd Films, 162 (1988) 375

6 D L A Tjaden, personal communication

7 H J Lippmann and F Kuhrt, Der Geometrie-einfluss auf den Hall-effekt bei rechteckigen Halbletterplatten, $Z$ Naturforsch, $13 a$ (1958) 474

$8 \mathrm{H}$ Leeuwis, Effect of 'overlapping' voltage contacts in planar Hall transducers, Sensors und Actuaturs, 4 (1983) 17-24

9 D A Thompson, L T Romankıw and A F Mayadas, Thın film magnetoresistors in memory, storage, and related applications, JEEE Trans Magn, MAG-11 (1975) 1039 
10 A H Morrish, The Physical Princuples of Magnetusm, Wiley, New York, 1965, p 11

$11 \mathrm{~J} \mathrm{H} \mathrm{J} \mathrm{Fluttman,} \mathrm{The} \mathrm{influence} \mathrm{of} \mathrm{sample} \mathrm{geometry} \mathrm{on} \mathrm{the}$ magnetoresistance of $\mathrm{N} 1 \mathrm{Fe}$ films, Thun Soldd Futms, 16 (1973) 269-276

12 R M de Ridder and J H Fluitman, Modelling the (galvano-) magnetıc behaviour of permalloy sensors, $J$ Phys (Parts) Colloq, 46 (1985) C6/287-C6/290

$13 \mathrm{H}$ A M van den Berg, Micromagnetıcs and domains on softferromagnetic media, Ph $D$ Thesis, University of Delft, Delft, The Netherlands, 1984

$14 \mathrm{~J} \mathrm{H}$ Flutman, Applicability of the planar Hall effect, $J$ Appl Phys, 52 (1981) 2468-2470

$15 \mathrm{C}$ Tsang, Magnetics of small magnetoresistive sensors, $J$ Appl Phys, 55 (1984) 2226

I6 W Kwiatkowskı and S Tumansk1, The permalloy magnetoresistive sensors-properties and applications, $J$ Phys $E$ Sci $\quad J$ strum, 19 (1986) 502

17 K E Kuujk, W J van Gestel and F W Gorter, The barber pole, a linear magnetoresistive head, IEEE Trans Magn, MAG-11 (1975) 1215

18 M Kitada, Y Kamo, $H$ Tanabe, $H$ Tsuchyla and $\mathrm{K}$ Momata, Magnetoresistive thin-film sensor with permanent magnet biasing film, $J$ Appl Phys, 58 (1985) 1667

19 F B Shelledy and G W Brock, A linear self-biased magnetoresistive head, IEEE Trans Magn, MAG-1I (1975) 1206

$20 \mathrm{~F}$ Jeffers, Soft-adjacent-layer self-biases magnetoresıstive heads in high-density recording, IEEE Trans Magn, MAG-2I (1985) 1563

$21 \mathrm{R}$ Ilunt, A magnetoresistive readout transdueer, IEEE Trans Magn, MAG-7 (1971) 150

$22 \mathrm{R}$ W Wood, Magnetic megabits, IEEE Spectrum, (May) (1990) 32-38

$23 \mathrm{~J}$ P J Groenland and J H J Fluttman, Measurement system for two-dimensional magnetic field distributions, applied to the investigation of recording head fields, $J$ Phys $E$ Scl Instrum, 14 (1981) 503-508

$24 \mathrm{~T}$ Wielınga, J H J Flustman and J C Lodder, Perpendicular stand-still recording in CoCr films, IEEE Trans Magn, MAG-19 (1983) 94

$25 \mathrm{~J}$ H J Fluitman, Recording head field measurement with a magnetoresıstuve transducer, IEEE Trans Magn, MAG-14 (1978) 433-435

$26 \mathrm{~J}$ H J Flustman and $\mathrm{H} \mathrm{W}$ Krabbe, Minature sensor for two-dimensional magnetıc field distributions $J$ Phys $E$ Sct Instrum, 5 (1972) 963

27 J H W Kuntzel, M G J Heijman and V Zieren, Technology for a submicron vertical magnetoresistive sensor, in J C Lodder (ed), Proc 3rd Sensors and Actuators Symp, Kluwer, Deventer, 1986, p 219

28 F G West, W J Odom, J A Ruce and T C Penn, Detection of low-intensity magnetic fields by means of ferromagnetic films, $J$ Appl Phys, 34 (1963) 1163-1164

29 R M de Ridder and J H Flutman, Orthogonal thin film magnetometer using the anisotropic magnetoresistance effect, IEEE Trans Magn, MAG-20 (1984) 690

$30 \mathrm{R}$ M de Rıdder, Thun-film magnetoresıstıve magnetometer, Ph $D$ Thests, University of Twente, Enschede, The Netherlands, 1988

31 F S Greene and R B Yarbrough, Orthogonal susceptıbility of permalloy films $J$ Appl Phys, 41 (1970) 4076-4082

$32 \mathrm{R} M$ de Rudder and J $H$ Fluitman, $A$ hysteresis model for an orthogonal thın-film magnetometer, IEEE Trans Magn, MAG-26 (1990) $1237-1245$

$33 \mathrm{R}$ Groothengel and $\mathbf{R} \mathrm{M}$ de Rudder, Thm-film magnetometer with integrated planar drive coll, in $\mathrm{J}$ C Lodder (ed), Proc 3rd Sensors and Actuators Symp, Kluwer, Deventer, 1986, p 211

$34 \mathrm{~K} J \mathbf{M}$ Eukel and $\mathrm{J} \quad \mathrm{H} \quad \mathrm{J}$ Flutman, Optımization of the response of magnetoresistive elements, IEEE Trans Magn, MAG26 (1990) $311-321$

$35 \mathrm{~K} J \mathbf{M}$ Eujkel, A thin-film magnetoresistive angle detector, $P h D$ Thests, University of Twente, Enschede, The Netherlands, 1988

36 3T Twente Technology Transfer BV, Enschede, The Netherlands, Contactless angle transducer, European Patent No 862016920 (1986)

37 J W Wieberdink and K J M Eukel, Permalloy multılayers to reduce the effects of uniaxial anisotropy, IEEE Trans Magn, MAG-25 (1989) 4278-4282

38 D W J Pulle, $\mathrm{K}$ J M Eıjkel, J H J Fluitman, $\mathrm{H}$ Leeuwis and D J $M$ van Mierlo, A new magneto-resistive based sensor for switched reluctance dnves, to be submitted to IEEE Trans Ind Appl

39 J P J Groenland, Thin-film magnetoresistive absolute position detector, Ph $D$ Thesis, University of Twente, Enschede, The Netheriands, 1990

$40 \mathrm{E} M$ Petriu, Absolute-type position transducers using a pseudorandom encodıng, IEEE Trans Instrum Meas, IM-36 (1987) 950-955

41 F A Pronk, J P J Groenland and T S J Lammennk, Simulation model for a silicon Hall sensor in an absolute digital position detection system, Solud-Siate Electron, 29 (1986) 579-584

\section{Biographies}

$J P J$ Groenland was born in Obdam, Netherlands, on June 7, 1950 He studied electrical eng1neering at the University of Twente, Enschede, Netherlands, where he recelved the M Sc degree in 1975 and the Ph D degree in 1990 Since 1975 he has been employed as a lecturer and research scientist at the University of Twente His interests now are in magnetic-recording principles

C $J M$ Eljkel was born in Hesloo, Netherlands, on October 1, $1959 \mathrm{He}$ recelved the M Sc degree in mathematics from the University of Amsterdam, Amsterdam, Netherlands, in 1983 and the Ph D degree from the University of Twente, Enschede, Netherlands, in 1988 Now he is head of the Clean-Room Laboratory for sensors and actuators at the University of Twente

J $H$ J Flutman was born in Beverw1jk, Netherlands, on March 23, 1938 He recelved the $M S c$ and $P h D$ degrees in physics from the University of Amsterdam, Amsterdam, Netherlands, in 1966 and 1970, respectively He joined 
the University of Twente, Enschede, Netherlands, in 1970, employed to teach applied magnetism and conducting research in the field of magnetic recording and magnetic transducers Since 1982 he has been full professor of transducer science and in 1990 he was appointed as scientific director of the MESA Research Institute at the University of Twente His interests now are in magnetic, optical and micromechanical principles
$R \quad M$ de Ridder was born in Amsterdam, Netherlands, on April 26, 1950 He studied electrical engineering at the University of Twente, Enschede, Netherlands, where he recelved the $M$ Sc degree in 1978 and the Ph D degree in 1988 Since 1981 he has been employed as a lecturer and research scientist at the University of Twente $\mathrm{H}_{1 \mathrm{~s}}$ interests now are in lightwave devices for optical telecommunication 